\title{
Stability and yield performance of different wheat genotypes across multi- locations of Khyber Pakhtunkhwa
}

\author{
Iltaf Ullah ${ }^{1}$, Haneef Raza ${ }^{1 *}$, Salman Ali ${ }^{1}$, Shah Fahad ${ }^{1}$, Adnan Ahmad ${ }^{1}$, \\ Sadia Mukhtiar ${ }^{1}$, Nadia Samad ${ }^{2}$ and Muhammad Babar ${ }^{3}$ \\ 1. Cereal Section Tarnab, Peshawar, Khyber Pakhtunkhwa Agricultural Research Department, Pakistan \\ 2. Horticulture Section Tarnab, Peshawar, Khyber Pakhtunkhwa Agricultural Research Department, Pakistan \\ 3. Department of Agriculture, University of Swabi-Anbar, Swabi-Khyber Pakhtunkhwa, Pakistan \\ *Corresponding author's email: rhanif5@gmail.com
}

Citation

Iltaf Ullah, Haneef Raza, Salman Ali, Shah Fahad, Adnan Ahmad, Sadia Mukhtiar, Nadia Samad and Muhammad Babar. Stability and yield performance of different wheat genotypes across multi locations of Khyber Pakhtunkhwa. Pure and Applied Biology. Vol. 11, Issue 3, pp793-805. http://dx.doi.org/10.19045/bspab.2022.110080

\begin{tabular}{llll}
\hline \hline Received: 02/08/2021 & Revised: 23/10/2021 & Accepted: 30/11/2021 & Online First: 07/12/2021
\end{tabular}

\section{Abstract}

Knowledge of genotype by environment interaction (GEI) is essential for identifying environment-specific and widely adapted genotypes. Multi-environment trials were conducted to evaluate the extent of genotype (G), environment (E) and GEI effects on grain yield of wheat. Thirty wheat genotypes were evaluated under five environments within Khyber Pakhtunkhwa, Pakistan during 2017/18 cropping seasons. Combined ANOVA for $G$ and GEI were significant for almost all traits. Significant GEI suggested that the performance of genotypes was not consistent across environments, revealing strong influence of environments. Generally, GEI explained major portion of total variation and thus had larger effect than $\mathrm{G}$ and $\mathrm{E}$ on the expression of phenotype of wheat yield per hectare. In present study, the contribution to total sum of squares, regardless of trait, revealed that environments (11.51 to $96.27 \%)$ and genotypes (0.98 to $13.28 \%$ ) play major role in managing the given traits. However, shares of genotype $\times$ environment and experimental error were 1.63 to $34.24 \%$ and 0.93 to $39.65 \%$, respectively. Regarding genotypic performance, the genotype WADAAN-17 (4330.80 kg per hectare) revealed maximum grain yield, whereas the minimum was exhibited by the genotype 25 TH SAWYT 305 (2837.50 kg per hectare). In case of genotype $\times$ environment interactions, maximum desirable seed yield was produced by genotype WADAAN-17 (5666.7 $\left.\mathrm{kg} \mathrm{ha}^{-1}\right)$ at Mansehra, genotype HYT 65 (S) E-51 (5091.7 kg ha-1) at CCRI, genotype HYT $70(\mathrm{~N})$ E-40 (2308.3 $\left.\mathrm{kg} \mathrm{ha}^{-1}\right)$ at Buner, genotype 38TH ESWYT 138(5625.0 kg ha-1) at Tarnab and genotype HYT $70(\mathrm{~N})$ E-16 (4879.2 $\left.\mathrm{kg} \mathrm{ha}^{-1}\right)$ at DI Khan. According to Eberhart and Russell (1966) stability model none of the genotype were found to show ideal stability $\left(S^{2} \mathrm{di}=0\right)$. However, based on their conditions genotypes HYT 65 (S) E-51, KHAISTA-17, 38TH ESWYT 110 and HYT 70 (N) E-1 were identified as relatively stable for grain yield. Considering the Wricke'secovalence (Wi) model genotypes 38 TH ESWYT 103, 38 TH ESWYT 102, 38TH ESWYT 106, HYT 70 (N) E-1, 38TH ESWYT 140, PASEENA-17, 25th SAWYT 307 and KHAISTA-17 showed better stability across the locations. Both Eberhart and Russell and Wricke'secovalence stability models similarly described the stability of 38TH ESWYT 110 and KHAISTA-17 for grain yield. Therefore, these genotypes could be used in wheat improvement program for enhancing stability.

Keywords: Genotype $\times$ environmental interaction, Stability in wheat genotypes, Grain yield 


\section{Introduction}

Wheat is the primary food source of people in Pakistan. At national level, wheat was grown on 9.2 million hectares which produced 25.1 million tons with an average yield of 2.7tonsha- ${ }^{-1}$, while in Khyber Pakhtunkhwa, it was grown on 0.73 million hectares which produced 1.2 million tons with an average yield 1.7 tonsha $^{-1}$ (Pak. Beurau Stat. 2014-15) [1]. The average national wheat yield of Pakistan is far below than agro-technologically advanced countries of the world. In Khyber Pakhtunkhwa province the situation is even worse where yield is lower than the national average yield. Factors for lower grain yield include lack of irrigation water, inadequate rainfall, heat stress, unavailability of high yielding cultivars and absence of quality seed. Demand for wheat being a staple food crop, would rise with the increase in population. Wheat production could be increased either by developing high yielding cultivars or growing more area under cultivation. The choice for wheat cultivation on larger area is partial; however, development of high yielding wheat cultivars with wider adaptability would play significant role.

Cultivars performance largely depends on their genetic makeup $(\mathrm{G})$, environment $(\mathrm{E})$ and their interaction (GEI). Fluctuating response of genotypes across test environments is a usual phenomenon, known as GEI $[2,3]$. Yield potential of a cultivar is the result of its performance over locations and years. Therefore, stability analysis of genotypes is required in the presence of GEI to ascertain high yielding and relatively

Table 1. Agro-metrological features of tested environments including temperature, rainfall

\begin{tabular}{|c|c|c|c|c|c|}
\hline \multirow{2}{*}{ Environments } & \multirow{2}{*}{$\begin{array}{c}\text { Growin } \\
\text { g season }\end{array}$} & \multicolumn{2}{|c|}{ Geographical position } & \multirow{2}{*}{$\begin{array}{c}\text { Altitude } \\
\text { (m.a.s.l) }\end{array}$} & \multirow{2}{*}{$\begin{array}{c}\text { Averagerainfal } \\
\text { I (mm) }\end{array}$} \\
\cline { 3 - 4 } & & Latitude & Longitude & & 376 \\
\hline Mansehra & $2017 / 18$ & $34.3313^{\circ} \mathrm{N}$ & $73.1980^{\circ} \mathrm{E}$ & 915 & 220 \\
\hline CCRI & $2017 / 18$ & $34.0159^{\circ} \mathrm{N}$ & $71.9755^{\circ} \mathrm{E}$ & 288 & 248 \\
\hline Buner & $2017 / 18$ & $34.4694^{\circ} \mathrm{N}$ & $72.6333^{\circ} \mathrm{E}$ & 944 & 403.9 \\
\hline Tarnab & $2017 / 18$ & $34.0162^{\circ} \mathrm{N}$ & $71.7056^{\circ} \mathrm{E}$ & 299 & 268.8 \\
\hline DI Khan & $2017 / 18$ & $31.8626^{\circ} \mathrm{N}$ & $70.9019^{\circ} \mathrm{E}$ & 165 & \\
\hline
\end{tabular}




\section{Experimental design and procedure}

Thirty genotypes including Waadan-17, Khaista-17 and Paseena-17 were field-tested in five environments during 2017/18. Experimental materials were planted in RCB design with two replicates at each environment. Standard dose of nitrogen (120 $\mathrm{kg} \mathrm{ha}^{-1}$ ) and phosphorous (80 $\mathrm{kg} \mathrm{ha}^{-1}$ ) was applied. Uniform cultural practices required for wheat crop were followed throughout the growing season.

\section{Traits measurement}

Data were recorded on days to heading, days to maturity, plant height and yield per hectare following the procedure described by [6].

\section{Statistical analysis}

\section{Analysis of variance}

Data recorded on various traits were statistically analyzed across five environments appropriate for RCBD design using SAS computer program [7]. Upon significant differences, means were separated using LSD test at 5\% level of probability.

\section{Results and Discussion}

\section{Analysis of variance}

Significant differences were observed among genotypes, environments (locations) and $\mathrm{G} \times \mathrm{E}$ interaction (GEI) means for all the traits, which assured greater genetic variability among the genotypes which might be due to divergent genetic makeup of the genotypes, as well as environments (Table 2). These results are in conformity with the findings of [8, 9]. Contrarily, Khan et al. [10] reported non-significant differences among genotypes, environments and their interaction for days to heading and for grain yield in wheat. In this respect, contrary observations may be due to differences in genetic makeup of the genotypes being studied, environmental factors or even both. The presence of $\mathrm{G} \times \mathrm{E}$ interaction makes necessary the evaluation of stability for grain yield traits to obtain good efficiency in breeding programs focused on development of high yielding lines [11]. In present study, the contribution to total sum of squares, regardless of trait, revealed that environments (11.51 to 96.27\%) and genotypes $(0.98$ to $13.28 \%$ ) play major role in managing the given traits. However, shares of genotype $\times$ environment and experimental error (replications) were 1.63 to $34.24 \%$ and 0.93 to $39.65 \%$, respectively.

Genotypes contribution was maximum for plant height (13.28\%), followed by grain yield per hectare $(7.67 \%)$, days to heading $(3.37 \%)$ and days to maturity (0.98\%) (Table $2)$. The highest share of the environment in the total variation was observed for days to maturity $(96.27 \%)$, followed by days to heading $(90.27 \%)$, grain yield per hectare $(62.29 \%)$ and plant height $(11.51 \%)$. Accumulation of $\mathrm{G} \times \mathrm{E}$ effects was highest for plant height $(34.24 \%)$, grain yield per hectare (13.93\%), days to heading (3.02\%), and days to maturity $(1.63 \%)$.

\section{Days to maturity(No.)}

For days to maturity, genotypic and genotype $\times$ location interactions mean values ranged from 163 to 168 days and 142 to 188 days, respectively (Table 3 ). The genotype $25 \mathrm{TH}$ SAWYT 307 and 25 TH SAWYT 305 revealed the minimum days to maturity with the mean value of 163 days each. The later genotype was again followed by three other genotypes viz., 38TH ESWYT 138, 38TH ESWYT 104 and 38TH ESWYT 103 with the mean value of 164 days each. However, the maximum days to maturity were taken by KT-17 and HYT $70(\mathrm{~N})$ E-1 with the mean value of 168 and 167 days, respectively. Considering locations means, on average the genotypes exhibited minimum days to maturity at DI Khan (142 days), followed by CCRI (161 days), Mansehra and Tarnab (162 days each) and Buner (182 days).

In case of genotype $\times$ environment interactions, it was evident that the genotype 25 TH SAWYT 305 and 25 TH SAWYT 307 revealed minimum days to maturity with a mean value of 142 days each at DI Khan 
followed by $25 \mathrm{TH}$ SAWYT 305 with the mean value of 161 days. While at Mansehra, 25 TH SAWYT 343 and 38TH ESWYT 116 exhibited minimum days to maturity with the mean value of 162 days to maturity each. The same days to maturity were taken by $25 \mathrm{TH}$ SAWYT 307 and 38TH ESWYT 138 at Tarnab. However, the genotypes HYT 70 (N) E-40, 38TH ESWYT 138, 25 TH SAWYT 305 and 25 TH SAWYT 307 manifested maximum days to maturity, each with a mean value of 185 days.

\section{Days to heading(No.)}

For days to maturity, genotypic and genotype $\times$ location interactions mean values ranged from 123 to 130 days and 108 to 142 days, respectively (Table 4). The genotype $25 \mathrm{TH}$ SAWYT and 25 TH SAWYT 305307 took minimum days to heading with the mean value of 123 and 124 days, respectively. Whereas, the maximum days to heading were taken by the check cultivar viz., KT-17 and Paseena-17 with the mean value of 130 days each. Considering locations means, on average the genotypes exhibited minimum days to heading at DI Khan (108 days), followed by Manshera (108 days), Tarnab (124 days), CCRI (127 days) and Buner (138 days) (Table 4).

In case of genotype $\times$ environment interactions, the minimum days to heading (108 days) were taken by the genotype $38 \mathrm{TH}$ ESWYT 103 at DI Khan, followed by 38TH ESWYT 138 at Mansherawith the mean value of 120 days. Similarly, two other genotypes viz., 25 TH SAWYT 307 and 38TH ESWYT 138 were earlier in heading at Tarnab with a mean value of 124 days. While at CCRI the genotypes i.e.,25 TH SAWYT 305, 25 TH SAWYT 307 and HYT 65 (S)E9 took minimum days to heading (127 days) and Buner (138 days). Whereas, across all these tested located Buner took maximum days with a mean value of 138 days for majority of the genotypes (Table 4).

\section{Plant height(cm)}

For plant height, genotypic and genotype $x$ location interactions mean values ranged from 88 to $100 \mathrm{~cm}$ and 71 to $115 \mathrm{~cm}$, respectively (Table 5). The genotype WADAAN-17 obtained maximum plant height $(100 \mathrm{~cm})$ followed by HYT $70(\mathrm{~N})$ E$40(99 \mathrm{~cm})$ and 25 TH SAWYT $324(97 \mathrm{~cm})$. Considering locations means, on average the genotypes exhibited maximum plant height at Buner $(115 \mathrm{~cm})$, followed by Tarnab $(108 \mathrm{~cm})$, Manshera $(101 \mathrm{~cm})$, CCRI and DI Khan $(99 \mathrm{~cm})$ each (Table 5).

In case of genotype $\times$ environment interactions, the maximum plant height was obtained by WAADAN-17 (115cm) followed by 38TH ESWYT $102(106 \mathrm{~cm})$ at Buner. At Tarnab the maximum plant height was observed by the genotype KT-17 followed by 38TH ESWYT 116, HYT 70 (N) E-40 and WADAAN-17. These four genotypes exhibited a mean value of $108 \mathrm{~cm}$. The genotypes HYT 70 (N) E-40, WADAAN-17 and KT-17 revealed maximum plant height at Mansehra with a mean value of $101 \mathrm{~cm}$. While at CCRI and DI Khan the genotypes 25 TH SAWYT 305 and 38TH ESWYT 140 revealed a maximum value of $99 \mathrm{~cm}$ each (Table 5).

\section{Grain yield per hectare}

Yield improvement is one of the major objectives of every plant breeding program and is a complex quantitative trait that is controlled by genetic potential and also influenced greatly by environmental factors. Means for seed yield of 30 wheat genotypes across five environments are given in (Table 6). Overall means ranged from 2837.5 to $4330.8 \mathrm{~kg} \mathrm{ha}^{-1}$. Fifteen out of 30 genotypes produced higher seed yield than averaged seed yield over all environments. Minimum seed yield across five environments was produced by genotypes 25th SAWYT 305 (2837.5 kg ha-1), followed by 25th SAWYT 343 (2980.8 $\mathrm{kg} \mathrm{ha}^{-1}$ ) and HYT 65 (S)E-34 (3000.8 $\left.\mathrm{kg} \mathrm{ha}^{-1}\right)$, whereas maximum seed 
yield (4330.8 $\mathrm{kg} \mathrm{ha}^{-1}$ ) across all environments was obtained from genotype 38TH ESWYT 103, followed by HYT 65 (S) E-51 (4172.5 $\mathrm{kg} \mathrm{ha}^{-1}$ ) and KHAISTA-17 (4039.8 kg ha-1). Among locations, the most productive environment for seed yield was Tarnab, produced an average seed yield of $4607.1 \mathrm{~kg}$ $\mathrm{ha}^{-1}$, followed by Mansehra (4294.3 $\left.\mathrm{kg} \mathrm{ha}^{-1}\right)$, DI Khan (3716.3 $\mathrm{kg} \mathrm{ha}^{-1}$ ) and CCRI (3622.2 $\mathrm{kg} \mathrm{ha}^{-1}$ ), while least productive environment for seed yield was Buner (1503.6 $\mathrm{kg} \mathrm{ha}^{-1}$ ). Maximum desirable seed yield was produced by genotype WADAAN-17 (5666.7 $\left.\mathrm{kg} \mathrm{ha}^{-1}\right)$ at Mansehra, genotype HYT 65 (S) E-51 (5091.7 $\mathrm{kg} \mathrm{ha}^{-1}$ ) at CCRI, genotype HYT 70 (N) E-40 (2308.3 $\left.\mathrm{kg} \mathrm{ha}^{-1}\right)$ at Buner, genotype $38 \mathrm{TH}$ ESWYT $138\left(5625.0 \mathrm{~kg} \mathrm{ha}{ }^{-1}\right)$ at Tarnab, and genotype HYT 70 (N) E-16 (4879.2 $\mathrm{kg} \mathrm{ha}^{-1}$ ) at DI Khan (Table 6).

\section{Stability analysis}

\section{Eberhart and Russell analysis}

Selection of desirable genotypes become difficult when evaluated across environments, because the presence of significant GEI reduce the relationship between genotypic and phenotypic values, thus preventing the expected genetic progression in breeding program. Therefore, stability study must be carried to identify genotypes with constant mean efficiency. Data of tested genotypes for grain yield were subjected to stability models that proposed by using [12]. According to this model, genotypes with high mean yield, having regression, coefficient (bi) approximating unity and minimum deviation from regression $\left(\mathrm{S}^{2} \mathrm{di}\right)$ will be stated as stable. Mean yield, coefficient of regression (bi) and deviation from regression $\left(\mathrm{S}^{2} \mathrm{di}\right)$ of 30 wheat genotypes across five environments are given in (Table $7 \& 8$ ). None of the genotype were found to show ideal stability $\left(S^{2} \mathrm{di}=0\right)$. Even though, based on given mean grain yield, coefficient of regression near to unity and having minimum deviation from regression genotype HYT 65 (S) E-51 (bi= 0.92 and
$\left.\mathrm{S}^{2} \mathrm{~d}_{\mathrm{i}}=39587.73\right)$ followed by KHAISTA-17 $\left(\mathrm{bi}=1.06\right.$ and $\left.\mathrm{S}^{2} \mathrm{di}=-41653.94\right), 38 \mathrm{TH}$ ESWYT $110\left(\mathrm{bi}=1.09\right.$ and $\left.\mathrm{S}^{2} \mathrm{di}=-31036.77\right)$ and HYT $70(\mathrm{~N})$ E-1 (bi= 1.07 and $\mathrm{S}^{2} \mathrm{di}=$ 167932.65) were identified as relatively stable genotypes that rank $2^{\text {nd }}, 3^{\text {rd }}, 4^{\text {th }}$ and $6^{\text {th }}$ for grain yield in (Table 9). The first genotype had regression coefficient (bi) values less than unity, which indicates that this is specifically suited to unfavorable environments. However, the remaining three genotypes had regression coefficient (bi) values greater than unity implied the wide adaptability of these genotypes to favorable environments. Our results are in accordance ofEberhart and Russell [13] who reported stable genotype for grain yield plant ${ }^{-1}$ in their study by using Eberhart and Russell model.

\section{Wricke'secovalence analysis}

Wricke'secovalence (Wi) is another model used as stability parameter. According to this model the genotypes with lowest $\mathrm{W} i$ value will be consider more stable compared to other genotypes. Wricke'secovalence was calculated for each of the 30 wheat genotypes evaluated across five diverse locations for one year in the major wheat growing regions of Khyber Pakhtunkhwa (Table 10). The genotypes with the lowest ecovalence contributed the least to the genotype by environment interaction and are therefore more stable. Accordingly, 38TH ESWYT 103, 38TH ESWYT 102, 38TH ESWYT 106, HYT 70 (N) E-1, 38TH ESWYT 140, PASEENA-17, 25th SAWYT 307 and KHAISTA-17 were the most stable genotypes that for grain yield ranked $14^{\text {th }}$, $10^{\text {th }}, 12^{\text {th }}, 06^{\text {th }}, 20^{\text {th }}, 13$ th, 25 th and 3 rd respectively. Although, the most unstable genotypes were HYT $70(\mathrm{~N})$ E-16, 38TH ESWYT 124, KT-17, HYT 65 (S) E-9, 38TH ESWYT 142, HYT 65 (S)E-8, HYT 65 (S) E5 and 25th SAWYT 325; that for grain yield ranked 15th, 16th, 18th, 21st, 27th, 22nd, 27 th and $23^{\text {rd }}$, respectively. The results indicate that some of the high yielding 
genotypes have lowest ecovalence and therefore, could be recommended for general adaptability.

Table 2. Mean squares of different wheat genotypes tested across five environments of Khyber Pakhtunkhwa during 2018-19

\begin{tabular}{|c|c|c|c|c|c|}
\hline Source & DF & Days to heading & Days to maturity & Plant height & Grain yield \\
\hline Environments & 4 & $\begin{array}{c}5417.05^{* *} \\
(90.27 \%)\end{array}$ & $\begin{array}{c}11925.47^{* *} \\
(96.27 \%)\end{array}$ & $\begin{array}{c}499.81^{*} \\
(11.51 \%)\end{array}$ & $\begin{array}{c}88378605.70^{* *} \\
(62.29 \%)\end{array}$ \\
\hline Reps w/n Loc & 5 & $\begin{array}{c}41.01^{* *} \\
(0.85 \%)\end{array}$ & $\begin{array}{c}18.50^{* *} \\
(0.19 \%)\end{array}$ & $\begin{array}{c}45.65^{\mathrm{NS}} \\
(1.31 \%)\end{array}$ & $\begin{array}{c}226532.67^{\mathrm{NS}} \\
(0.20 \%)\end{array}$ \\
\hline Genotypes & 29 & $\begin{array}{c}27.91 * * \\
(3.37 \%)\end{array}$ & $\begin{array}{c}16.75^{* *} \\
(0.98 \%)\end{array}$ & $\begin{array}{c}79.55^{*} \\
(13.28 \%)\end{array}$ & $\begin{array}{c}1500989.88^{* *} \\
(7.67 \%)\end{array}$ \\
\hline G $\times$ L & 116 & $\begin{array}{c}6.25 * \\
(3.02 \%)\end{array}$ & $\begin{array}{c}6.96 * * \\
(1.63 \%)\end{array}$ & $\begin{array}{c}51.27^{* *} \\
(34.24 \%)\end{array}$ & $\begin{array}{c}681522.25^{* *} \\
(13.93 \%)\end{array}$ \\
\hline Pooled Error & 145 & $\begin{array}{c}4.10 \\
(2.48 \%)\end{array}$ & $\begin{array}{c}3.19 \\
(0.93 \%)\end{array}$ & $\begin{array}{c}47.49 \\
(39.65 \%)\end{array}$ & $\begin{array}{c}622798.77 \\
(15.91 \%)\end{array}$ \\
\hline CV $(\%)$ & - & 1.59 & 1.08 & 7.35 & 22.24 \\
\hline
\end{tabular}

$*, * *=$ Significant at $1 \%$ and 5\% level of probability, N.S = Non-significant, DF = Degree of freedom, CV = coefficient of variation and ()$=$ values in parenthesis are percent sum squares

Table 3. Means for days to maturity of different genotypes evaluated across five environments during 2018-19

\begin{tabular}{|c|c|c|c|c|c|c|}
\hline \multirow{2}{*}{ Genotypes } & \multicolumn{4}{|c|}{ Days to maturity } & \multirow[b]{2}{*}{ DI Khan } & \multirow{2}{*}{$\begin{array}{c}\text { Genotype } \\
\text { means }\end{array}$} \\
\hline & Mansehra & CCRI & Buner & Tarnab & & \\
\hline 25 TH SAWYT 305 & 165 & 161 & 185 & 163 & 142 & 163 \\
\hline 25 TH SAWYT 307 & 165 & 162 & 185 & 162 & 142 & 163 \\
\hline 25 TH SAWYT 324 & 164 & 165 & 187 & 167 & 145 & 165 \\
\hline 25 TH SAWYT 325 & 166 & 162 & 186 & 164 & 145 & 165 \\
\hline 25 TH SAWYT 343 & 162 & 163 & 186 & 167 & 146 & 165 \\
\hline 38 TH ESWYT 102 & 163 & 165 & 187 & 170 & 151 & 167 \\
\hline 38 TH ESWYT 103 & 166 & 162 & 186 & 165 & 142 & 164 \\
\hline 38 TH ESWYT 104 & 163 & 163 & 186 & 164 & 144 & 164 \\
\hline 38 TH ESWYT 106 & 167 & 165 & 187 & 168 & 145 & 166 \\
\hline 38 TH ESWYT 110 & 163 & 163 & 186 & 166 & 146 & 165 \\
\hline 38 TH ESWYT 111 & 165 & 163 & 186 & 167 & 150 & 166 \\
\hline 38 TH ESWYT 140 & 167 & 164 & 186 & 167 & 150 & 166 \\
\hline 38 TH ESWYT 116 & 162 & 164 & 186 & 166 & 149 & 165 \\
\hline 38 TH ESWYT 124 & 164 & 163 & 186 & 167 & 148 & 165 \\
\hline 38 TH ESWYT 138 & 164 & 162 & 185 & 162 & 147 & 164 \\
\hline 38 TH ESWYT 142 & 166 & 164 & 187 & 166 & 144 & 165 \\
\hline 38 TH ESWYT 145 & 165 & 163 & 188 & 168 & 153 & 167 \\
\hline 39 TH ESWYT 108 & 164 & 164 & 187 & 164 & 146 & 165 \\
\hline HYT 65 (S) E-5 & 169 & 162 & 186 & 165 & 144 & 165 \\
\hline HYT 65 (S)E-8 & 163 & 163 & 186 & 168 & 145 & 165 \\
\hline HYT 65 (S)E-9 & 165 & 163 & 186 & 169 & 148 & 166 \\
\hline HYT $65(\mathrm{~S}) \mathrm{E}-34$ & 168 & 162 & 186 & 165 & 143 & 165 \\
\hline
\end{tabular}




\begin{tabular}{|c|c|c|c|c|c|c|}
\hline HYT 65 (S) E-51 & 164 & 162 & 188 & 170 & 152 & 167 \\
\hline HYT 70 (N) E-1 & 166 & 164 & 188 & 170 & 150 & 167 \\
\hline HYT 70 (N) E-16 & 167 & 162 & 186 & 167 & 146 & 165 \\
\hline HYT 70 (N) E-40 & 166 & 164 & 185 & 168 & 145 & 165 \\
\hline WADAAN-17 & 163 & 165 & 188 & 170 & 148 & 167 \\
\hline KHAISTA-17 & 165 & 164 & 185 & 170 & 151 & 167 \\
\hline PASEENA-17 & 164 & 163 & 188 & 169 & 153 & 167 \\
\hline KT-17 & 165 & 163 & 188 & 171 & 153 & 168 \\
\hline Loc means & 165 & 163 & 186 & 167 & 147 & 165 \\
\hline
\end{tabular}

$\mathrm{LSD}_{0.05}$ Genotypes $=1.58$, Locations $=0.64, \mathrm{GLI}=3.53$

Table 4. Means for days to heading of different genotypes evaluated across five environments during 2018-19

\begin{tabular}{|c|c|c|c|c|c|c|}
\hline \multirow{2}{*}{ Genotypes } & \multicolumn{4}{|c|}{ Days to heading } & \multirow[b]{2}{*}{ DI Khan } & \multirow{2}{*}{ Genotype means } \\
\hline & Mansehra & CCRI & Buner & Tarnab & & \\
\hline 25 TH SAWYT 305 & 121 & 127 & 138 & 125 & 108 & 124 \\
\hline 25 TH SAWYT 307 & 121 & 127 & 138 & 124 & 108 & 123 \\
\hline 25 TH SAWYT 324 & 126 & 129 & 139 & 129 & 111 & 127 \\
\hline 25 TH SAWYT 325 & 128 & 129 & 138 & 126 & 111 & 126 \\
\hline 25 TH SAWYT 343 & 126 & 129 & 138 & 129 & 112 & 127 \\
\hline 38 TH ESWYT 102 & 128 & 128 & 142 & 132 & 117 & 129 \\
\hline 38 TH ESWYT 103 & 125 & 128 & 138 & 127 & 108 & 125 \\
\hline 38 TH ESWYT 104 & 128 & 129 & 139 & 126 & 110 & 126 \\
\hline 38 TH ESWYT 106 & 129 & 128 & 142 & 130 & 111 & 128 \\
\hline 38 TH ESWYT 110 & 128 & 129 & 140 & 128 & 112 & 127 \\
\hline 38 TH ESWYT 111 & 127 & 129 & 138 & 129 & 116 & 128 \\
\hline 38 TH ESWYT 140 & 126 & 128 & 138 & 129 & 116 & 127 \\
\hline 38 TH ESWYT 116 & 124 & 130 & 139 & 128 & 115 & 127 \\
\hline 38 TH ESWYT 124 & 127 & 128 & 140 & 129 & 114 & 127 \\
\hline 38 TH ESWYT 138 & 120 & 130 & 138 & 124 & 113 & 125 \\
\hline 38 TH ESWYT 142 & 126 & 130 & 141 & 128 & 110 & 127 \\
\hline 38 TH ESWYT 145 & 128 & 129 & 142 & 130 & 119 & 129 \\
\hline 39 TH ESWYT 108 & 130 & 129 & 141 & 126 & 112 & 127 \\
\hline HYT 65 (S) E-5 & 126 & 129 & 139 & 127 & 110 & 126 \\
\hline HYT 65 (S)E-8 & 127 & 128 & 140 & 130 & 111 & 127 \\
\hline HYT 65 (S)E-9 & 127 & 127 & 138 & 131 & 114 & 127 \\
\hline HYT 65 (S)E-34 & 127 & 128 & 138 & 127 & 109 & 126 \\
\hline HYT 65 (S) E-51 & 127 & 129 & 142 & 132 & 118 & 129 \\
\hline HYT $70(\mathrm{~N})$ E-1 & 127 & 130 & 142 & 132 & 116 & 129 \\
\hline HYT $70(\mathrm{~N})$ E-16 & 126 & 129 & 138 & 129 & 112 & 127 \\
\hline HYT $70(\mathrm{~N})$ E-40 & 126 & 129 & 138 & 130 & 111 & 126 \\
\hline WADAAN-17 & 128 & 129 & 141 & 132 & 114 & 128 \\
\hline KHAISTA-17 & 127 & 129 & 140 & 132 & 117 & 129 \\
\hline PASEENA-17 & 128 & 130 & 141 & 131 & 119 & 130 \\
\hline KT-17 & 128 & 128 & 142 & 133 & 119 & 130 \\
\hline Loc means & 126 & 128 & 139 & 129 & 113 & 127 \\
\hline
\end{tabular}

$\mathrm{LSD}_{0.05}$ Genotypes $=1.79$, Locations $=0.73, \mathrm{GLI}=4.0$ 
Table 5. Means for plant height of different genotypes evaluated across five environments during 2018-19

\begin{tabular}{|c|c|c|c|c|c|c|}
\hline \multirow{2}{*}{ Genotypes } & \multicolumn{4}{|c|}{ Plant height (cm) } & \multirow[b]{2}{*}{ DI Khan } & \multirow{2}{*}{ Genotype means } \\
\hline & Mansehra & CCRI & Buner & Tarnab & & \\
\hline 25 TH SAWYT 305 & 92 & 99 & 98 & 95 & 93 & 95 \\
\hline 25 TH SAWYT 307 & 94 & 91 & 96 & 98 & 93 & 94 \\
\hline 25 TH SAWYT 324 & 93 & 91 & 105 & 98 & 98 & 97 \\
\hline 25 TH SAWYT 325 & 87 & 95 & 99 & 90 & 92 & 92 \\
\hline 25 TH SAWYT 343 & 96 & 90 & 100 & 105 & 91 & 96 \\
\hline 38 TH ESWYT 102 & 100 & 93 & 106 & 100 & 84 & 97 \\
\hline 38 TH ESWYT 103 & 90 & 92 & 93 & 98 & 89 & 92 \\
\hline 38 TH ESWYT 104 & 95 & 95 & 94 & 98 & 86 & 93 \\
\hline 38 TH ESWYT 106 & 87 & 90 & 91 & 95 & 98 & 92 \\
\hline 38 TH ESWYT 110 & 98 & 95 & 90 & 93 & 96 & 94 \\
\hline 38 TH ESWYT 111 & 95 & 87 & 94 & 93 & 93 & 92 \\
\hline 38 TH ESWYT 140 & 87 & 89 & 77 & 93 & 99 & 89 \\
\hline 38 TH ESWYT 116 & 99 & 88 & 105 & 105 & 87 & 97 \\
\hline 38 TH ESWYT 124 & 97 & 83 & 71 & 100 & 90 & 88 \\
\hline 38 TH ESWYT 138 & 96 & 86 & 94 & 98 & 97 & 94 \\
\hline 38 TH ESWYT 142 & 90 & 88 & 87 & 90 & 93 & 90 \\
\hline 38 TH ESWYT 145 & 97 & 90 & 89 & 98 & 98 & 94 \\
\hline 39 TH ESWYT 108 & 96 & 94 & 95 & 95 & 92 & 94 \\
\hline HYT 65 (S) E-5 & 89 & 91 & 88 & 95 & 91 & 91 \\
\hline HYT 65 (S)E-8 & 99 & 83 & 96 & 91 & 93 & 92 \\
\hline HYT 65 (S)E-9 & 94 & 84 & 96 & 100 & 88 & 92 \\
\hline HYT 65 (S)E-34 & 91 & 89 & 91 & 98 & 92 & 92 \\
\hline HYT 65 (S) E-51 & 91 & 86 & 87 & 103 & 90 & 91 \\
\hline HYT $70(\mathrm{~N})$ E-1 & 100 & 91 & 91 & 93 & 92 & 93 \\
\hline HYT $70(\mathrm{~N})$ E-16 & 93 & 91 & 103 & 95 & 93 & 95 \\
\hline HYT $70(\mathrm{~N})$ E-40 & 101 & 92 & 103 & 105 & 97 & 99 \\
\hline WADAAN-17 & 101 & 89 & 115 & 105 & 92 & 100 \\
\hline KHAISTA-17 & 95 & 91 & 101 & 103 & 91 & 96 \\
\hline PASEENA-17 & 95 & 93 & 93 & 100 & 97 & 95 \\
\hline KT-17 & 101 & 92 & 93 & 108 & 88 & 96 \\
\hline Loc means & 94 & 90 & 95 & 98 & 92 & 94 \\
\hline
\end{tabular}

LSD $_{0.05}$ Genotypes $=6.09$, Locations $=2.49, \mathrm{GLI}=13.62$

Table 6. Means for grain yield of different genotypes evaluated across five environments during 2018-19

\begin{tabular}{|c|c|c|c|c|c|c|}
\hline \multirow{2}{*}{ Genotypes } & \multicolumn{4}{|c|}{${\text { Grain yield }\left(\mathbf{k g ~ h a c}^{-\mathbf{1}}\right)}^{*} \begin{array}{c}\text { Genotype } \\
\text { means }\end{array}$} \\
\cline { 2 - 7 } & Mansehra & CCRI & Buner & Tarnab & DI Khan & 2837.5 \\
\hline 25 TH SAWYT 305 & 3708.3 & 2025.0 & 1425.0 & 4166.7 & 2862.5 & 3065.6 \\
\hline 25 TH SAWYT 307 & 4032.1 & 2616.7 & 1550.0 & 3895.8 & 3233.3 & 3140.8 \\
\hline 25 TH SAWYT 324 & 4083.3 & 3800.0 & 1216.7 & 4104.2 & 2500.0 & 3157.5 \\
\hline 25 TH SAWYT 325 & 3875.0 & 2150.0 & 1354.2 & 4229.2 & 4179.2 & 2980.8 \\
\hline 25 TH SAWYT 343 & 3583.3 & 2383.3 & 1541.7 & 4458.3 & 2937.5 & 3759.2 \\
\hline 38 TH ESWYT 102 & 4791.7 & 3775.0 & 1620.8 & 4687.5 & 3920.8 & 3656.7 \\
\hline 38 TH ESWYT 103 & 4666.7 & 3633.3 & 1566.7 & 4583.3 & 3833.3 & \\
\hline
\end{tabular}




\begin{tabular}{|c|c|c|c|c|c|c|}
\hline 38 TH ESWYT 104 & 4666.7 & 3816.7 & 1470.8 & 3520.8 & 3933.3 & 3481.7 \\
\hline 38 TH ESWYT 106 & 4458.3 & 3566.7 & 1350.0 & 5041.7 & 4195.8 & 3722.5 \\
\hline 38 TH ESWYT 110 & 5025.0 & 4366.7 & 1816.7 & 5208.3 & 3333.3 & 3950.0 \\
\hline 38 TH ESWYT 111 & 4916.7 & 3566.7 & 1800.0 & 5604.2 & 3425.0 & 3862.5 \\
\hline 38 TH ESWYT 140 & 4229.2 & 3800.0 & 1250.0 & 4854.2 & 3083.3 & 3443.3 \\
\hline 38 TH ESWYT 116 & 3937.5 & 4933.3 & 2079.2 & 4645.8 & 3829.2 & 3885.0 \\
\hline 38 TH ESWYT 124 & 5500.0 & 2983.3 & 1129.2 & 4958.3 & 3145.8 & 3543.3 \\
\hline 38 TH ESWYT 138 & 4083.3 & 4083.3 & 1579.2 & 5625.0 & 3879.2 & 3850.0 \\
\hline 38 TH ESWYT 142 & 2833.3 & 3891.7 & 1579.2 & 3387.5 & 3412.5 & 3020.8 \\
\hline 38 TH ESWYT 145 & 4437.5 & 3150.0 & 958.3 & 3991.7 & 4745.8 & 3456.7 \\
\hline 39 TH ESWYT 108 & 5062.5 & 4100.0 & 962.5 & 4958.3 & 4208.3 & 3858.3 \\
\hline HYT 65 (S) E-5 & 2958.3 & 3758.3 & 1454.2 & 4583.3 & 2445.8 & 3040.0 \\
\hline HYT 65 (S)E-8 & 3437.5 & 2791.7 & 1191.7 & 5229.2 & 4325.0 & 3395.0 \\
\hline HYT 65 (S)E-9 & 3604.2 & 2983.3 & 1550.0 & 4104.2 & 4875.0 & 3423.3 \\
\hline HYT 65 (S)E-34 & 3583.3 & 2308.3 & 1150.0 & 4708.3 & 3254.2 & 3000.8 \\
\hline HYT 65 (S) E-51 & 4729.2 & 5091.7 & 2195.8 & 5062.5 & 3783.3 & 4172.5 \\
\hline HYT 70 (N) E-1 & 4208.3 & 4091.7 & 1583.3 & 5041.7 & 4483.3 & 3881.7 \\
\hline HYT 70 (N) E-16 & 3333.3 & 4291.7 & 1379.2 & 4125.0 & 4879.2 & 3601.7 \\
\hline HYT 70 (N) E-40 & 4375.0 & 4475.0 & 2308.3 & 4416.7 & 3212.5 & 3757.5 \\
\hline WADAAN-17 & 5666.7 & 4341.7 & 1404.2 & 5479.2 & 4762.5 & 4330.8 \\
\hline KHAISTA-17 & 5166.7 & 4658.3 & 1833.3 & 4895.8 & 3641.7 & 4039.2 \\
\hline PASEENA-17 & 4333.3 & 4433.3 & 1562.5 & 4354.2 & 3745.8 & 3685.8 \\
\hline KT-17 & 5541.7 & 2800.0 & 1245.8 & 4291.7 & 3420.8 & 3460.0 \\
\hline Loc means & 4294.3 & 3622.2 & 1503.6 & 4607.1 & 3716.3 & 3548.7 \\
\hline
\end{tabular}

$\mathrm{LSD}_{0.05}$ Genotypes $=697.55$, Locations $=284.77, \mathrm{GLI}=1559.77$

Table 7. Mean, regression coefficient (bi) and deviation from regression $\left(s^{2} d i\right)$ values for plant height and seed yield of different wheat genotypes evaluated across five environments during 2018-19

\begin{tabular}{|c|c|c|c|c|c|c|}
\hline \multirow{2}{*}{ Genotypes } & \multicolumn{3}{|c|}{ Plant height } & \multicolumn{3}{c|}{ Grain yield per hectare } \\
\cline { 2 - 7 } & Mean & Bi & S $^{\mathbf{2}} \mathbf{i}$ & Mean & Bi & $\mathbf{S}^{\mathbf{2} \text { di }}$ \\
\hline 25th SAWYT 305 & 95.3 & -0.29 & -13.38 & 2837.50 & 0.83 & 63230.63 \\
\hline 25th SAWYT 307 & 94.2 & 0.84 & -22.86 & 3065.58 & 0.80 & -174554.56 \\
\hline 25th SAWYT 324 & 96.8 & 0.88 & 8.76 & 3140.82 & 0.95 & 36692.32 \\
\hline 25th SAWYT 325 & 92.2 & -0.43 & 1.75 & 3157.51 & 0.93 & 330297.92 \\
\hline 25th SAWYT 343 & 96.4 & 2.08 & -19.28 & 2980.84 & 0.83 & -13844.48 \\
\hline 38TH ESWYT 102 & 96.5 & 1.68 & 38.27 & 3759.16 & 1.04 & -278176.82 \\
\hline 38TH ESWYT 103 & 92.2 & 0.84 & -16.73 & 3656.69 & 1.02 & -279649.87 \\
\hline 38TH ESWYT 104 & 93.1 & 0.75 & -2.89 & 3481.67 & 0.87 & 117140.19 \\
\hline 38TH ESWYT 106 & 92.2 & 0.19 & 0.78 & 3722.51 & 1.17 & -257796.69 \\
\hline 38TH ESWYT 110 & 94 & -0.41 & -12.95 & 3950.01 & 1.09 & -31036.77 \\
\hline 38TH ESWYT 111 & 92.1 & 0.70 & -17.27 & 3862.51 & 1.15 & 1705.72 \\
\hline 38TH ESWYT 140 & 88.7 & -0.30 & 58.68 & 3443.33 & 1.11 & -165715.58 \\
\hline 38TH ESWYT 116 & 96.7 & 2.72 & 1.11 & 3884.99 & 0.78 & 137001.81 \\
\hline 38TH ESWYT 124 & 88 & 1.68 & 124.63 & 3543.34 & 1.32 & 302588.92 \\
\hline 38TH ESWYT 138 & 93.8 & 1.29 & -11.36 & 3850.00 & 1.14 & -59530.62 \\
\hline 38TH ESWYT 142 & 89.5 & 0.07 & -16.14 & 3020.84 & 0.56 & 115919.68 \\
\hline 38TH ESWYT 145 & 94 & 0.68 & -3.62 & 3456.68 & 1.12 & 305965.92 \\
\hline
\end{tabular}




\begin{tabular}{|c|c|c|c|c|c|c|}
\hline 39TH ESWYT 108 & 94.3 & 0.26 & -21.91 & 3858.34 & 1.37 & -245040.91 \\
\hline HYT 65 (S) E-5 & 90.6 & 0.41 & -15.40 & 3040.00 & 0.81 & 326083.42 \\
\hline HYT 65 (S)E-8 & 92.1 & 1.15 & 11.36 & 3395.00 & 1.12 & 381188.70 \\
\hline HYT 65 (S)E-9 & 92.2 & 2.19 & -22.10 & 3423.34 & 0.83 & 433849.05 \\
\hline HYT 65 (S)E-34 & 92 & 0.94 & -19.41 & 3000.84 & 1.02 & 47648.79 \\
\hline HYT 65 (S) E-51 & 91.3 & 1.88 & -4.92 & 4172.49 & 0.92 & 39587.73 \\
\hline HYT 70 (N) E-1 & 93.2 & 0.28 & -7.52 & 3881.68 & 1.07 & -167932.65 \\
\hline HYT 70 (N) E-16 & 95 & 0.71 & -0.02 & 3601.66 & 0.88 & 644503.73 \\
\hline HYT 70 (N) E-40 & 99.2 & 1.79 & -21.81 & 3757.50 & 0.69 & -12045.19 \\
\hline WADAAN-17 & 100.1 & 2.67 & 48.43 & 4330.83 & 1.40 & -228819.26 \\
\hline KHAISTA-17 & 95.9 & 1.76 & -15.07 & 4039.17 & 1.06 & -41653.94 \\
\hline PASEENA-17 & 95.3 & 0.74 & -16.80 & 3685.82 & 0.95 & -105083.69 \\
\hline KT-17 & 96 & 2.24 & 3.73 & 3460.00 & 1.17 & 445809.19 \\
\hline
\end{tabular}

Table 8. Mean, regression coefficient (bi) and deviation from regression $\left(\mathrm{s}^{2} \mathrm{di}\right)$ values for days to heading and maturity of different wheat genotypes evaluated across five environments during 2018-19

\begin{tabular}{|c|c|c|c|c|c|c|}
\hline \multirow{2}{*}{ Genotypes } & \multicolumn{3}{|c|}{ Days to heading } & \multicolumn{3}{c|}{ Days to maturity } \\
\cline { 2 - 7 } & Mean & Bi & $\mathbf{S}^{\mathbf{2}} \mathbf{d i}$ & Mean & Bi & $\mathbf{S}^{\mathbf{2}} \mathbf{~}$ \\
\hline 25 TH SAWYT 305 & 123.5 & -0.52 & 1.11 & 163.1 & 1.08 & 2.79 \\
\hline 25 TH SAWYT 307 & 123.3 & 0.71 & 1.13 & 162.7 & 1.09 & 1.78 \\
\hline 25 TH SAWYT 324 & 126.5 & -0.76 & 1.07 & 165.3 & 1.07 & 0.37 \\
\hline 25 TH SAWYT 325 & 126.2 & 1.24 & 1.01 & 164.5 & 1.03 & 0.53 \\
\hline 25 TH SAWYT 343 & 126.6 & -0.46 & 0.99 & 164.5 & 1.03 & 0.63 \\
\hline 38 TH ESWYT 102 & 129.1 & 2.43 & 0.93 & 167 & 0.92 & 3.16 \\
\hline 38 TH ESWYT 103 & 125.1 & -0.69 & 1.14 & 164.1 & 1.10 & 1.77 \\
\hline 38 TH ESWYT 104 & 126.1 & 3.18 & 1.10 & 163.8 & 1.06 & 0.37 \\
\hline 38TH ESWYT 106 & 127.7 & 1.09 & 1.13 & 166.2 & 1.05 & 1.09 \\
\hline 38TH ESWYT 110 & 127.3 & -0.40 & 1.04 & 164.7 & 1.01 & -1.10 \\
\hline 38TH ESWYT 111 & 127.6 & -1.95 & 0.83 & 166 & 0.91 & -0.60 \\
\hline 38TH ESWYT 140 & 127.1 & -1.67 & 0.84 & 166.4 & 0.92 & -0.88 \\
\hline 38TH ESWYT 116 & 126.7 & 0.88 & 0.91 & 165.2 & 0.95 & 1.51 \\
\hline 38TH ESWYT 124 & 127.3 & -1.75 & 0.99 & 165.3 & 0.97 & -1.22 \\
\hline 38TH ESWY 138 & 124.6 & 10.41 & 0.95 & 163.8 & 0.97 & 1.80 \\
\hline 38TH ESWYT 142 & 126.9 & -0.88 & 1.17 & 165.1 & 1.08 & 0.13 \\
\hline 38TH ESWYT 145 & 129.3 & 1.90 & 0.86 & 167.1 & 0.91 & 3.82 \\
\hline 39TH ESWYT 108 & 127.4 & 4.28 & 1.07 & 164.9 & 1.03 & 0.35 \\
\hline HYT 65 (S) E-5 & 125.9 & -0.55 & 1.11 & 164.9 & 1.06 & 7.41 \\
\hline HYT 65 (S)E-8 & 126.9 & -0.71 & 1.11 & 164.6 & 1.05 & 0.22 \\
\hline HYT 65 (S)E-9 & 127.2 & 0.08 & 0.91 & 165.9 & 0.97 & -0.36 \\
\hline HYT 65 (S)E-34 & 125.6 & 0.39 & 1.09 & 164.7 & 1.07 & 6.49 \\
\hline HYT 65 (S) E-51 & 129.3 & 2.50 & 0.89 & 167 & 0.93 & 6.46 \\
\hline HYT 70 (N) E-1 & 129 & -0.30 & 1.00 & 167.3 & 0.98 & -0.43 \\
\hline HYT 70 (N) E-16 & 126.6 & -0.46 & 0.99 & 165.3 & 1.02 & 0.87 \\
\hline HYT 70 (N) E-40 & 126.4 & 0.61 & 1.03 & 165.3 & 1.01 & 1.76 \\
\hline WADAAN-17 & 128.4 & -0.81 & 1.02 & 166.6 & 1.03 & 2.22 \\
\hline KHAISTA-17 & 128.8 & -0.14 & 0.87 & 166.7 & 0.88 & 1.26 \\
\hline PASEENA-17 & 129.5 & -0.35 & 0.84 & 167.3 & 0.91 & 4.42 \\
\hline
\end{tabular}




\begin{tabular}{|l|l|l|l|l|l|l|} 
KT-17 & 129.9 & 3.66 & 0.87 & 167.8 & 0.92 & 5.71 \\
\hline
\end{tabular}

Table 9. Mean, regression coefficient (bi) and deviation from regression $\left(s^{2} d i\right)$ values for Grain yield of different wheat genotypes evaluated across five environments during 2018-19

\begin{tabular}{|c|c|c|c|c|}
\hline \multirow{2}{*}{ Genotypes } & \multicolumn{4}{|c|}{ Grain yield $\left(\mathbf{K g ~ h a c}^{-\mathbf{1}}\right)$} \\
\cline { 2 - 5 } & $\mathbf{B i}$ & $\mathbf{S}^{\mathbf{2}} \mathbf{\text { i }}$ & Mean & Rank \\
\hline 25th SAWYT 305 & 0.83 & 63230.63 & 2837.50 & 30 \\
\hline 25th SAWYT 307 & 0.80 & -174554.56 & 3065.58 & 25 \\
\hline 25th SAWYT 324 & 0.95 & 36692.32 & 3140.82 & 24 \\
\hline 25th SAWYT 325 & 0.93 & 330297.92 & 3157.51 & 23 \\
\hline 25th SAWYT 343 & 0.83 & -13844.48 & 2980.84 & 29 \\
\hline 38TH ESWYT 102 & 1.04 & -278176.82 & 3759.16 & 10 \\
\hline 38TH ESWYT 103 & 1.02 & -279649.87 & 3656.69 & 14 \\
\hline 38TH ESWYT 104 & 0.87 & 117140.19 & 3481.67 & 17 \\
\hline 38TH ESWYT 106 & 1.17 & -257796.69 & 3722.51 & 12 \\
\hline 38TH ESWYT 110 & 1.09 & -31036.77 & 3950.01 & 04 \\
\hline 38TH ESWYT 111 & 1.15 & 1705.72 & 3862.51 & 07 \\
\hline 38TH ESWYT 140 & 1.11 & -165715.58 & 3443.33 & 20 \\
\hline 38TH ESWYT 116 & 0.78 & 137001.81 & 3884.99 & 05 \\
\hline 38TH ESWYT 124 & 1.32 & 302588.92 & 3543.34 & 16 \\
\hline 38TH ESWYT 138 & 1.14 & -59530.62 & 3850.00 & 09 \\
\hline 38TH ESWYT 142 & 0.56 & 115919.68 & 3020.84 & 27 \\
\hline 38TH ESWYT 145 & 1.12 & 305965.92 & 3456.68 & 19 \\
\hline 39TH ESWYT 108 & 1.37 & -245040.91 & 3858.34 & 08 \\
\hline HYT 65 (S) E-5 & 0.81 & 326083.42 & 3040.00 & 26 \\
\hline HYT 65 (S)E-8 & 1.12 & 381188.70 & 3395.00 & 22 \\
\hline HYT 65 (S)E-9 & 0.83 & 433849.05 & 3423.34 & 21 \\
\hline HYT 65 (S)E-34 & 1.02 & 47648.79 & 3000.84 & 28 \\
\hline HYT 65 (S) E-51 & 0.92 & 39587.73 & 4172.49 & 02 \\
\hline HYT 70 (N) E-1 & 1.07 & -167932.65 & 3881.68 & 06 \\
\hline HYT 70 (N) E-16 & 0.88 & 644503.73 & 3601.66 & 15 \\
\hline HYT 70 (N) E-40 & 0.69 & -12045.19 & 3757.50 & 11 \\
\hline WADAAN-17 & 1.40 & -228819.26 & 4330.83 & 01 \\
\hline KHAISTA-17 & 1.06 & -41653.94 & 4039.17 & 03 \\
\hline PASEENA-17 & 0.95 & -105083.69 & 3685.82 & 13 \\
\hline KT-17 & 1.17 & 445809.19 & 3460.00 & 18 \\
\hline
\end{tabular}

Table 10. Wricke'secovalence value for 30 wheat genotypes evaluated across five locations

\begin{tabular}{|c|c|c|c|c|}
\hline Genotypes & Wi & Rank & Grain yield(kgha $\left.{ }^{-1}\right)$ & Rank \\
\hline 25th SAWYT 305 & 1294547.5 & 13 & 2837.5 & 30 \\
\hline 25th SAWYT 307 & 654125.0 & 24 & 3065.6 & 25 \\
\hline 25th SAWYT 324 & 1058890.6 & 18 & 3140.8 & 24 \\
\hline 25th SAWYT 325 & 1957611.1 & 08 & 3157.5 & 23 \\
\hline 25th SAWYT 343 & 1058005.2 & 19 & 2980.8 & 29 \\
\hline 38TH ESWYT 102 & 111300.1 & 29 & 3759.2 & 10 \\
\hline 38TH ESWYT 103 & 98765.6 & 30 & 3656.7 & 14 \\
\hline
\end{tabular}




\begin{tabular}{|c|c|c|c|c|}
\hline 38TH ESWYT 104 & 1382161.3 & 12 & 3481.7 & 17 \\
\hline 38TH ESWYT 106 & 321394.1 & 28 & 3722.5 & 12 \\
\hline 38TH ESWYT 110 & 889049.6 & 21 & 3950.0 & 04 \\
\hline 38TH ESWYT 111 & 1064945.5 & 17 & 3862.5 & 07 \\
\hline 38TH ESWYT 140 & 506270.2 & 26 & 3443.3 & 20 \\
\hline 38TH ESWYT 116 & 1626194.9 & 10 & 3885.0 & 05 \\
\hline 38TH ESWYT 124 & 2450795.1 & 02 & 3543.3 & 16 \\
\hline 38TH ESWYT 138 & 871597.8 & 22 & 3850.0 & 09 \\
\hline 38TH ESWYT 142 & 2399120.1 & 05 & 3020.8 & 27 \\
\hline 38TH ESWYT 145 & 1937261.5 & 09 & 3456.7 & 19 \\
\hline 39TH ESWYT 108 & 997380.2 & 20 & 3858.3 & 08 \\
\hline HYT 65 (S) E-5 & 2126440.1 & 07 & 3040.0 & 26 \\
\hline HYT 65 (S)E-8 & 2160577.7 & 06 & 3395.0 & 22 \\
\hline HYT 65 (S)E-9 & 2403594.4 & 04 & 3423.3 & 21 \\
\hline HYT 65 (S)E-34 & 1079847.1 & 16 & 3000.8 & 28 \\
\hline HYT 65 (S) E-51 & 1093714.6 & 15 & 4172.5 & 02 \\
\hline HYT 70 (N) E-1 & 457005.1 & 27 & 3881.7 & 06 \\
\hline HYT 70 (N) E-16 & 2957669.6 & 01 & 3601.7 & 15 \\
\hline HYT 70 (N) E-40 & 1453364.1 & 11 & 3757.5 & 11 \\
\hline WADAAN-17 & 1207296.3 & 14 & 4330.8 & 01 \\
\hline KHAISTA-17 & 829483.1 & 23 & 4039.2 & 03 \\
\hline PASEENA-17 & 633655.6 & 25 & 3685.8 & 13 \\
\hline KT-17 & 2445908.2 & 03 & 3460.0 & 18 \\
\hline
\end{tabular}

\section{Conclusion and Recommendation}

In case of genotype $x$ environment interactions, maximum desirable seed yield was produced by genotype WADAAN-17 at Mansehra, genotype HYT 65 (S) E-51 at CCRI, genotype HYT 70 (N) E-40 at Buner, the genotype 38TH ESWYT 138 at Tarnab and genotype HYT 70 (N) E-16 at DI Khan. According to [13] stability model none of the genotype were found to show ideal stability $\left(\mathrm{S}^{2} \mathrm{di}=0\right)$. However, based on their conditions genotypes HYT 65 (S) E-51, KHAISTA-17, 38TH ESWYT 110 and HYT 70 (N) E-1 were identified as relatively stable for grain yield. Considering the Wricke'secovalence (Wi) model genotypes 38 TH ESWYT 103, 38 TH ESWYT 102, 38TH ESWYT 106, HYT 70 (N) E-1, 38TH ESWYT 140, PASEENA-17, 25 TH SAWYT 307 and
KHAISTA-17 showed better stability across the locations. Both Eberhart and Russell and Wricke'secovalence stability models similarly described the stability of $38 \mathrm{TH}$ ESWYT 110and KHAISTA-17 for grain yield. Therefore, these genotypes could be used in wheat improvement program for enhancing stability.

\section{Authors' contributions}

Conceived and designed the experiments: I Ullah, Performed the experiments: S Ali \& S Fahad, Analyzed the data: A Ahmad, Contributed materials/ analysis/ tools: S Mukhtiar \& N Samad, Wrote the paper: H Raza \& M Babar.

\section{References}

1. Pakistan Bureau of Statistics (2014-15). Pakistan economic survey government of 
Pakistan, finance division, economic advisory wing, Islamabad.

2. Akcura M, Kaya Y \& Taner S (2009). Evaluation of durum wheat genotypes using parametric and nonparametric stability statistics. Turk J Field Crop 14(2): 111-122.

3. Mohammadi M, Karimizadeh R, Sabaghnia N \& Shefazadeh MK (2012). Genotype $\times$ environment interaction and yield stability analysis of new improved bread wheat genotypes. Turk J Field Crop 17(1): 67-73.

4. Annicchiarico P (2002). Genotype $\times$ environment interaction: challenges and opportunities for plant breeding and cultivar recommendations. (No. 174). Food and Agriculture Organization of the United Nations.

5. Mohammad F, Abdalla OS, Rajaram S, Yaljarouka A, Khan NU, Khan AZ, Khalil SK, Khalil IH, Ahmad I \& Jadoon SA (2011). Additive main effect and multiplicative analysis of syntheticderived wheat under varying moisture regimes. Pak J Bot 43(2): 1205-1210.

6. Sayre KD, Rajaram S \& Ficher RA (1997). Wheat yield potential in short bread wheat in North Mexico. Crop Sci 37(1): 36-42.

7. SAS Institute Inc (2009). SAS/STAT ® 9.2 User's Guide, Second Edition. Copyright (C) 2009, SAS. Inst. Inc. Cary, NC, USA. (https://support. sas.com/documentation/cdl/en/statug/63 033/ HTML/default/vie wer.htm).

8. Mehari M, Tesfay M, Yirga H, Mesele A, Abebe T, Workineh A \& Amare B (2015). GGEbiplot analysis of genotypeby-environment interaction and grain yield stability of bread wheat genotypes in South Tigray, Ethiopia. Commun. Biometry Crop. Sci 10(1): 17-26.

9. Ebrahimnejad S \& Rameeh V (2016). Correlation and factor analysis of grain yield and some important component characters in spring bread wheat genotypes. Cer Agron 1(165): 5-15.

10. Khan N, Syeed S, Masood A, Nazar R \& Iqbal N (2010). Application of salicylic acid increases contents of nutrients and antioxidative metabolism in mungbean and alleviates adverse effects of salinity stress. Inter J Plant Biol 1: 1-10.

11. Bilgin O, Guzmán C, Başer İ, Crossa J \& Korkut KZ (2016). Evaluation of grain yield and quality traits of bread wheat genotypes cultivated in Northwest Turkey. Crop Sci 56(1): 73-84.

12. Kaya Y, Palta C \& Taner S (2002). Additive main effects and multiplicative interaction analysis of yield performances in bread wheat genotypes across environments. Tur J Agric For 26(5): 275-279.

13. Eberhart ST \& Russell WA (1966). Stability parameters for comparing varieties 1. Crop Sci 6(1): 36-40. 